\title{
ESTUDO DA LIGAÇÃO CRUZADA INDUZIDA PELO FORMALDEÍDO EM CÁPSULAS DE GELATINA DURA
}

\author{
Study of formaldehyde induced cross-linking in hard gelatin capsules
}

\author{
Ana Lúcia T. C. Zampieri; Eliana M. Lima*, Danielle G. A. Diniz \\ Faculdade de Farmácia/ UFG - Laboratório de Tecnologia Farmacêutica/ FF \\ * Autor para correspondência: e-mail: emlima@farmacia.ufg.br
}

Recebido em 23/11/2005 - Aceito em 16/12/2005

\begin{abstract}
RESUMO: O formaldeído é um agente desnaturante que induz a ligação cruzada covalente na gelatina, sendo um dos agentes utilizados para a promoção da gastro-resistência em formas farmacêuticas gelatinosas, contudo, seu uso carece de avaliações minuciosas e da definição de protocolos específicos para as diversas classes de fármacos. O objetivo deste trabalho foi investigar a ligação cruzada induzida pelo formaldeído em cápsulas de gelatina dura. Cápsulas foram imersas em solução hidroalcoólica (70-90\%) contendo diversas concentrações de formaldeído. Amostras foram submetidas aos testes de avaliação da extensão da ligação cruzada determinandose o número de grupos amino livres por espectrofotometria e a quantificação do formaldeído residual feita por HPLC. A extensão da ligação cruzada ocorrida foi notavelmente influenciada pela concentração de formaldeído e de água e álcool nas soluções usadas para o tratamento das cápsulas. Os resultados demonstraram que o resíduo de formaldeído determinado após o tratamento das cápsulas esteve dentro de limites aceitáveis. Entretanto, avaliações seriadas durante o armazenamento das cápsulas demonstraram que a ligação cruzada continua ocorrendo, levando a uma resistência superior ao necessário para a administração de fármacos nesta forma farmacêutica.
\end{abstract}

PALAVRAS-CHAVE: gastro-resistência; cápsulas de gelatina; formaldeído; ligação-cruzada.

ABSTRACT: Formaldehyde induced cross-linking in gelatin can be used to promote gastric-resistance in gelatin based oral drug delivery systems. However, thorough evaluations for each drug class must be performed before establishing its use for this purpose. In this work, formaldehyde induced cross-linking in hard gelatin capsules was investigated. Capsules were immersed in hydroalcoholic (70-90\%) solutions containing several different concentrations of formaldehyde. Cross-linking was evaluated by the determination of free amino groups in the samples by UV spectrophotometry. Residual formaldehyde was determined by HPLC. Cross-linking reaction was strongly influenced by water and alcohol concentrations in the solutions used for the treatment of the capsules. Residual formaldehyde was within acceptable range. However, repeated analysis during a short term storage of the capsules proved that the cross-linking continues as long as any residual formaldehyde is present, leading to a much higher resistance than what would be convenient for the oral drug delivery.

KEYWORDS: gastric-resistance; gelatin capsules; formaldehyde; cross-linking.

\section{INTRODUÇÃO}

As formas farmacêuticas sólidas de administração oral representam a grande maioria dos produtos farmacêuticos disponíveis no mercado. É importante destacar que, embora essas formas farmacêuticas sejam absorvidas no trato gastrintestinal, há regiões ao longo deste sistema onde certos fármacos são melhor absorvidos. Por exemplo, existem fármacos que por serem irritantes ao estômago ou sensíveis ao baixo valor do pH do suco gástrico (entre 1,0 e 2,5) devem passar intactos pelo estômago para serem absorvidos no intestino. Isso é possível protegendo a forma farmacêutica com revestimentos ditos gastro-resistentes (PRISTA et al, 1995). 
Zampieri, A.L.T.C., et al./Revista Eletrônica de Farmácia Vol 2 (2), 73-79, 2005.

O revestimento gastro-resistente é uma técnica utilizada na preparação de formas farmacêuticas para que resistam, sem alteração, à ação do suco gástrico, devendo, porém, desagregar-se rapidamente no suco intestinal (TRONDSTAD et al,1985; RADI \& AMIJI, 2003) e tem despertado o interesse de muitos pesquisadores em desenvolver métodos eficazes e reprodutíveis para esse revestimento.

Vários materiais e técnicas podem ser usados na preparação dos revestimentos gastro-resistentes, como por exemplo, PINA et al (1995 e 1996) usaram a técnica de imersão ou mergulho seguido de secagem na preparação de cápsulas de gelatina dura com soluções hidroalcoólicas de formaldeído tornando-as gastroresistentes. O formaldeído é um agente desnaturante que induz a ligação cruzada covalente na gelatina o que proporciona gastro-resistência e possibilita sua degradadação por ação de enzimas no intestino (PINA et al, 1995 e 1996). Esta reação é predominantemente devida à formação das bases de Schiff, reação entre os grupos amino da lisina ou grupos laterais da hidroxilisina da gelatina e o aldeído disponível (SCHACHT et al, 1997; FRIESS et al, 1998). A figura 1 representa a formação da base de Schiff resultante da ação do formaldeído.

$$
\mathrm{Gel}-\mathrm{NH}_{2}+\mathrm{CH}_{2} \mathrm{O} \longrightarrow \mathrm{Gel}-\mathrm{N}=\mathrm{CH}_{2}+\mathrm{H}_{2} \mathrm{O}
$$

Figura 1: Representação da formação da base de Schiff (SCHACHT et al, 1997).

Embora o formaldeído seja o agente de revestimento há mais tempo conhecido da tecnologia farmacêutica (BRÓJO \& SOUSA, 1972; PINA et al, 1995 e 1996; GHOLAP \& SINGH, 2004) seu uso no tratamento de cápsulas de gelatina com o objetivo de torná-las gastro-resistentes, carece de avaliações minuciosas e da definição de protocolos específicos para as diversas classes de fármacos.

Diversos parâmetros relacionados à ligação cruzada na gelatina induzida pelo formaldeído, para a obtenção de cápsulas gastro-resistentes têm sido estudados (BRÓJO \& SOUSA, 1972; PINA et al 1995 e 1996), como por exemplo, a concentração do formaldeído na solução gastro-resistente; o tempo de contato dessa solução com a cápsula de gelatina dura; os processos de secagem das cápsulas após o contato com a solução de tratamento; o tempo de estocagem dessas cápsulas e a possível presença de formaldeído residual nas cápsulas gastro-resistentes. Devido a essas variáveis, o tratamento gastro-resistente em cápsulas de gelatina dura apresenta dificuldades em sua realização. A investigação desses parâmetros para a criação de um método de tratamento torna-se imprescindível, caso contrário o processo pode ser considerado empírico (BRÓJO \& SOUSA, 1972). Estudar o efeito da extensão da ligação cruzada em cápsulas de gelatina dura contendo naproxeno, a partir de soluções de tratamento contendo diferentes concentrações de formaldeído e do etanol e determinar o resíduo de formaldeído nas cápsulas gastro-resistentes.

\section{MATERIAL E MÉTODOS}

\section{Materiais}

Foram empregados os seguintes materiais: cápsulas de gelatina dura, incolor, número zero, naproxeno, formaldeído $36,6 \%$, álcool etílico $96^{\circ} \mathrm{GL}$, álcool etílico P. A., ácido clorídrico, ácido fosfórico, hidróxido de sódio, fosfato monobásico de potássio, acetonitrila, 2,4-dinitrofenilhidrazina (2,4-DNPH), bicarbonato de sódio, ácido trinitrobenzenosulfônico (TNBS) e éter etílico. Os equipamentos usados foram Espectrofotômetro UV-Vis (VarianCary-50), HPLC (Varian, modelo Pro-Star 240), Agitador Magnético (Tecnal, modelo TE-085), Balança Analítica (Gehaka, modelo AG 200), Balança de Precisão (Gehaka, modelo BG 4001), Estufa de Secagem (Quimis ${ }^{\circledR}$ ) e Encapsulador Manual (Gehaka).

O fármaco Naproxeno utilizado como modelo no processo desenvolvido neste trabalho, é um agente antinflamatório não esteróide (AINES) derivado do ácido propiônico; com fórmula estrutural apresentada na figura 2. É praticamente insolúvel em água (THE MERCK INDEX, 2001) e apresenta pka igual a 4,15 (AHFS DRUG INFORMATION, 1996). É um efetivo agente analgésico, antinflamatório e anti-pirético. Apresenta baixa tolerância gástrica e diversos estudos demonstram que o naproxeno pode causar danos à mucosa gástrica podendo gerar ulceração e/ou hemorragia (TRONDSTAD et al, 1985; SHEBA et al, 2002). Com isso, a diminuição da irritação gástrica proveniente da administração de naproxeno tem sido estudada através de modificações na forma farmacêutica e administração do fármaco (TRONDSTAD et al, 1985; ODDSSON et al, 1990).<smiles>COc1ccc2cc(C(C)C(=O)O)ccc2c1</smiles>

Figura 2: Fórmula Estrutural do Naproxeno. 
Zampieri, A.L.T.C., et al./Revista Eletrônica de Farmácia Vol 2 (2), 73-79, 2005.

\section{Métodos}

\section{Preparação das cápsulas gastro-resistentes}

As cápsulas foram manipuladas contendo 250mg de naproxeno de acordo com as Boas Práticas de Manipulação (BRASIL-RDC $n^{\circ} 33,2000$ ) e seladas manualmente através da imersão da extremidade aberta da tampa das cápsulas em uma solução contendo glicerina, álcool etílico $96^{\circ} \mathrm{GL}$ e água, em partes iguais.

As soluções de formaldeído $\left(0,2 \mathrm{mMol}^{-1} \mathrm{~L}^{-1}\right.$ a $\left.0,5 \mathrm{mMol}^{-1} \mathrm{~L}^{-1}\right)$ foram preparadas em solução hidroalcoólica (70 a 95\% de etanol), com as quais foram tratados grupos de cápsulas de naproxeno contendo 12 unidades cada, através da imersão em $100 \mathrm{~g}$ de solução por 15 minutos, em frasco aberto (PINA et al, 1996). As cápsulas foram secadas inicialmente em estufa a $37^{\circ} \mathrm{C}$ por 30 minutos e em seguida foram expostas à temperatura ambiente por 24 horas.

\section{Avaliação da ligação cruzada}

O número de moles de grupos amino livres da gelatina antes e depois do tratamento com diversos aldeídos têm sido usados para determinar a extensão da ligação cruzada ocorrida (OFNER, et al 1996; VANDELLI et $\mathrm{al}, 2001,2004)$. Este teste foi realizado pesando uma quantidade exata de $11 \mathrm{mg}$ de cápsulas vazias previamente tratadas com diferentes soluções contendo formaldeído e etanol. Essas cápsulas foram tratadas com $1 \mathrm{ml}$ de bicarbonato de sódio por 04 horas a $40^{\circ} \mathrm{C}$ e $1 \mathrm{ml}$ de ácido trinitrobenzenosulfônico (TNBS). Foram adicionados $3 \mathrm{ml}$ de ácido clorídrico $6 \mathrm{~N}$ e o material foi autoclavado por 01 hora a $120^{\circ} \mathrm{C}(15-17 \mathrm{psi})$. O produto hidrolisado foi diluído com $5 \mathrm{ml}$ de água e extraído com $5 \mathrm{ml}$ de éter etílico. Uma alíquota de $5 \mathrm{ml}$ da fase aquosa foi aquecida a $37^{\circ} \mathrm{C}$ por 15 minutos, resfriada à temperatura ambiente e diluída novamente com $15 \mathrm{ml}$ de água. $\mathrm{O}$ número de moles dos grupos amino livres de gelatina foram determinados por espectrofotometria na região do UV em $346 \mathrm{~nm}$, contra um branco. A solução referência (branco) foi preparada da mesma forma que a amostra acima descrita, porém o ácido clorídrico $6 \mathrm{~N}$ foi adicionado antes do TNBS a fim de bloquear a reação com os grupos amino livres da gelatina.

Os cálculos foram realizados convertendo os valores das absorbâncias obtidas para moles de grupos amino por grama da gelatina, a partir do método descrito por OFNER et al (1996).

\section{Quantificação do formaldeído residual}

Para este teste foram avaliadas cápsulas previamente submetidas a diferentes tratamentos com concentrações variadas de formaldeído $\left(0,2-0,37 \mathrm{mMol}^{-1} \mathrm{~L}^{-1}\right)$ e de etanol nas soluções de tratamento $(70-90 \%)$. As amostras para o ensaio foram coletadas após 24 horas de exposição à temperatura ambiente, como também após 30 dias de armazenamento. Duas cápsulas foram depositadas em frasco fechado contendo $20 \mathrm{ml}$ de água destilada em temperatura ambiente e agitadas em agitador magnético por 2 horas. Posteriormente foi retirado 01 $\mathrm{ml}$ para análise em HPLC do formaldeído residual.

\section{Método para quantificação do formaldeído residual}

O método usado foi baseado no método proposto por HEYDEN et al (2002). Para a cromatografia líquida de alta eficiência foi utilizada coluna Licosphere RP-18 (250x4mm), tendo como fase móvel uma mistura de 45/55 ( $/ / v)$ de acetonitrila e $0,025 \mathrm{M}$ de solução tampão fosfato ajustado para pH 4,0 com uma solução $1 \mathrm{M}$ de ácido fosfórico. As condições de análise foram: fluxo $2 \mathrm{ml} / \mathrm{min}$, volume de injeção $20 \mu \mathrm{l}$, detecção ultravioleta $(\lambda=345 \mathrm{~nm})$.

Em etapa prévia ao ensaio cromatográfico foi promovida a derivação da amostra contendo formaldeído com 2,4-dinitrofenilhidrazina (2,4-DNPH), possibilitando assim, a quantificação do formaldeído, a partir do produto dessa reação. Nessa derivação $0,4 \mathrm{ml}$ de uma solução de 2,4 -DNPH $0,1 \%$ foi adicionada a 1,0ml de amostra. A mistura foi agitada por 01 minuto em vórtex seguido de repouso por 02 minutos. A solução foi estabilizada adicionando $0,4 \mathrm{ml}$ de uma solução tampão fosfato $0,1 \mathrm{M}(\mathrm{pH} 6,8)$ e $0,7 \mathrm{ml}$ de $\mathrm{NaOH} 1 M$. Esta mistura foi então injetada no cromatógrafo.

A curva de calibração foi preparada a partir de uma solução estoque de formaldeído $(0,1 \%(\mathrm{~m} / \mathrm{v}))$ em fase móvel. Pela regressão linear da curva, foi obtido um coeficiente de correlação igual a 0,8502. A expressão da curva foi Conc. $=Y-55210 / 8 * 10^{7}$.

Os cálculos referentes à concentração do formaldeído residual foram efetuados a partir da área dos picos identificados através da separação cromatográfica do produto de reação formado pelo formaldeído e o reagente 2,4-DNPH.

\section{RESULTADOS E DISCUSSÃO}

\section{Avaliação da ligação cruzada}

Os resultados referentes à avaliação da extensão da ligação cruzada ocorrida entre as moléculas de gelatina estão apresentados na figura 3 e indicaram a diminuição do número de moles dos grupos amino livres 
Zampieri, A.L.T.C., et al./Revista Eletrônica de Farmácia Vol 2 (2), 73-79, 2005.

após o tratamento com concentrações crescentes de formaldeído. Entretanto, quando a concentração de formaldeído foi constante e a concentração de etanol aumentou houve o aumento do número de moles dos grupos amino livres. Como esse número, após o tratamento com formaldeído, representam a extensão da ligação cruzada, é possível concluir que a ligação cruzada nas cápsulas gastro-resistentes tratadas com o formaldeído cresceu com o aumento da concentração de formaldeído e decresceu com o aumento da concentração de etanol.

Os resultados obtidos estão de acordo com VANDELLI et al (2001), que verificaram a extensão da ligação cruzada promovida pelo DL-gliceraldeído (GAL) em microesferas de gelatina. Foi verificado que o aumento na concentração de GAL promoveu a diminuição dos grupos amino livres, ou seja, o aumento da ligação cruzada. Da mesma maneira, VANDELLI et al (2004) verificaram a extensão da ligação cruzada, em microesferas de gelatina, promovida pelo aquecimento em fornos a diferentes temperaturas. Constataram que, o aumento na temperatura dos fornos promoveu a diminuição dos grupos amino livres, ou seja, o aumento da ligação cruzada.

Portanto, a variação da quantidade de grupos amino livre da gelatina evidenciou os diferentes níveis de ligação cruzada ocorrida após o tratamento com o formaldeído, tendo sido esta a reação responsável pela gastroresistência das cápsulas.

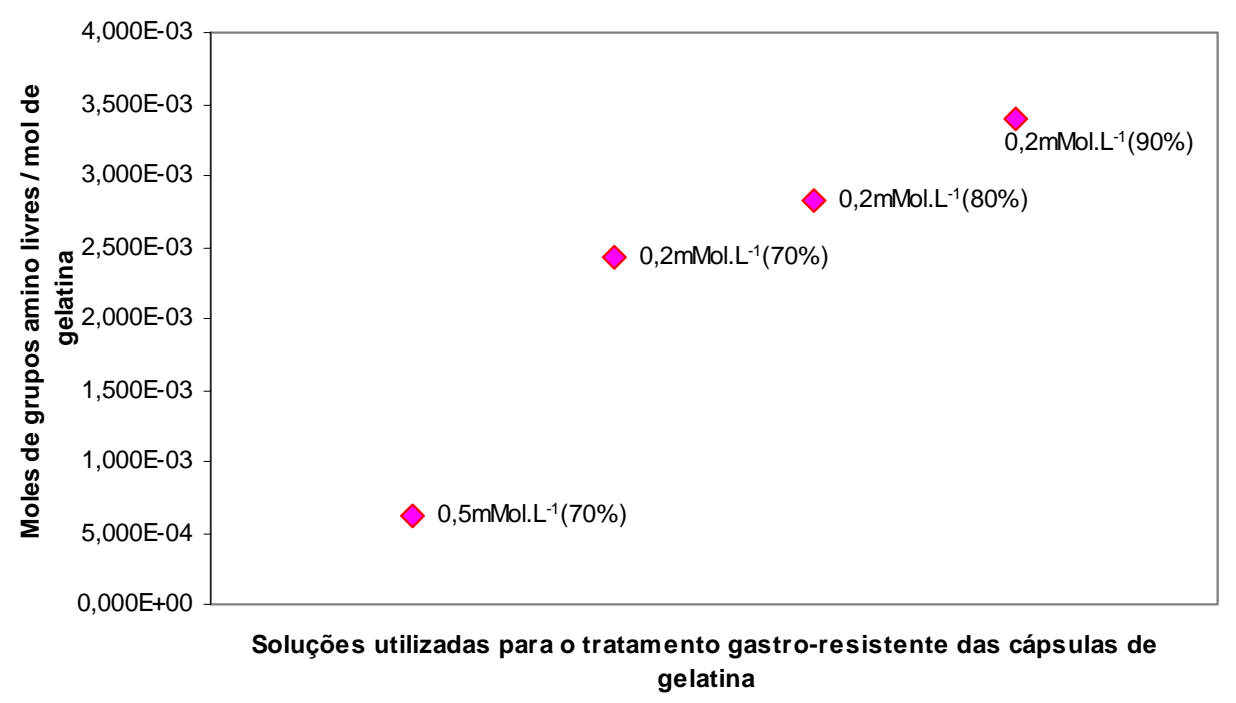

Figura 3: Relação entre moles dos grupos amino livres por moles de gelatina presentes nas cápsulas de gelatina dura após tratamento gastro-resistente com diferentes soluções contendo formaldeído $0,2-0,5 \mathrm{mMol}^{-1} \mathrm{~L}^{-1}$ e etanol 70 $90 \%$.

\section{Quantificação do formaldeído residual}

Os resultados obtidos estão apresentados na tabela 1 e demonstraram que, para as cápsulas analisadas após o procedimento de secagem e exposição à temperatura ambiente por 24 horas, quanto maior a concentração de formaldeído presente na solução de tratamento, maior a concentração residual. De maneira inversa, quanto maior a concentração de etanol na solução de tratamento menor o resíduo de formaldeído quantificado.

PINA et al (1987; 1996) avaliaram cápsulas de gelatina dura gastro-resistentes a partir da exposição destas em diferentes soluções contendo formaldeído e etanol e observaram que quanto maior a concentração de formaldeído maior era a concentração residual desse. Contudo, a concentração de formaldeído residual detectado por PINA et al (1987) nas cápsulas tratadas com 2,0\% de formaldeído em $90 \%$ de etanol foi 0,332mg/cápsula, ao passo que, a concentração obtida nesse trabalho, para as cápsulas tratadas com $0,2 \mathrm{mMol}^{-1} \mathrm{~L}^{-1}$ de formaldeído e $90 \%$ de etanol (concentração similar àquela citada por PINA et al (1987) foi 0,022 mg/cápsula. Essa diferença pode ser explicada pelas diferentes metodologias empregadas nos tratamentos gastro-resistentes, como por exemplo, o tamanho da cápsula usada, já que o trabalho citado usou uma cápsula bem maior (número 00). 
Zampieri, A.L.T.C., et al./Revista Eletrônica de Farmácia Vol 2 (2), 73-79, 2005.

Tabela 1 - Quantificação do Formaldeído residual após o procedimento de secagem e exposição à temperatura ambiente por 24 horas

\begin{tabular}{cc}
\hline Soluções de tratamento & Formaldeído residual mg/cápsula \\
\hline $0,37 \mathrm{mMol} . \mathrm{L}^{-1}$ de formaldeído / $70 \%$ de etanol & $0,536 \mathrm{mg} / \mathrm{cápsula}$ \\
$0,2 \mathrm{mMol} \mathrm{L}^{-1}$ de formaldeído / $70 \%$ de etanol & $0,360 \mathrm{mg} / \mathrm{cápsula}$ \\
$0,2 \mathrm{mMol} \mathrm{L}^{-1}$ de formaldeído / $80 \%$ de etanol & $0,041 \mathrm{mg} / \mathrm{cápsula}$ \\
$0,2 \mathrm{mMol} \mathrm{L}^{-1}$ de formaldeído / $90 \%$ de etanol & $0,022 \mathrm{mg} / \mathrm{cápsula}$ \\
\hline
\end{tabular}

Após 30 dias de armazenamento das cápsulas tratadas com $0,2 \mathrm{mMol} . \mathrm{L}^{-1}$ de formaldeído e $90 \%$ de etanol não foi detectada a presença de formaldeído residual demonstrando que, devido à sua volatilidade, o formaldeído residual diminui com tempo de armazenamento.

De acordo com INTERNATIONAL PROGRAMME ON CHEMICAL SAFETY (1989), a concentração de formaldeído normalmente ingerida por um adulto através dos alimentos pode variar de 1,5 a 14mg/dia. A partir dessa informação é possível concluir que, todas as cápsulas analisadas após 24 horas do tratamento apresentaram concentração residual dentro de limites aceitáveis, sendo que, nas cápsulas tratadas com $0,2 \mathrm{mMol} . \mathrm{L}^{-1}$ de formaldeído e $90 \%$ de etanol essa concentração foi ainda menos significativa

Os cromatogramas referentes à tabela 1 estão apresentados na figura 4. Esses cromatogramas demonstraram que o produto de reação do formaldeído foi mais evidente nas amostras de cápsulas tratadas com maior concentração de formaldeído presente nas soluções de tratamento, sendo, contudo, menos evidentes nas amostras de cápsulas tratadas com maior concentração de etanol. É importante ressaltar que a área do pico formado pelo produto de reação do formaldeído é inversamente proporcional à área do pico relativo ao reagente 2,4-DNPH.

Os resultados demonstraram também que as modificações propostas ao método de HEYDEN et al (2002), permitiram uma redução no tempo de eluição do composto analisado, como também uma melhor definição dos picos formados.

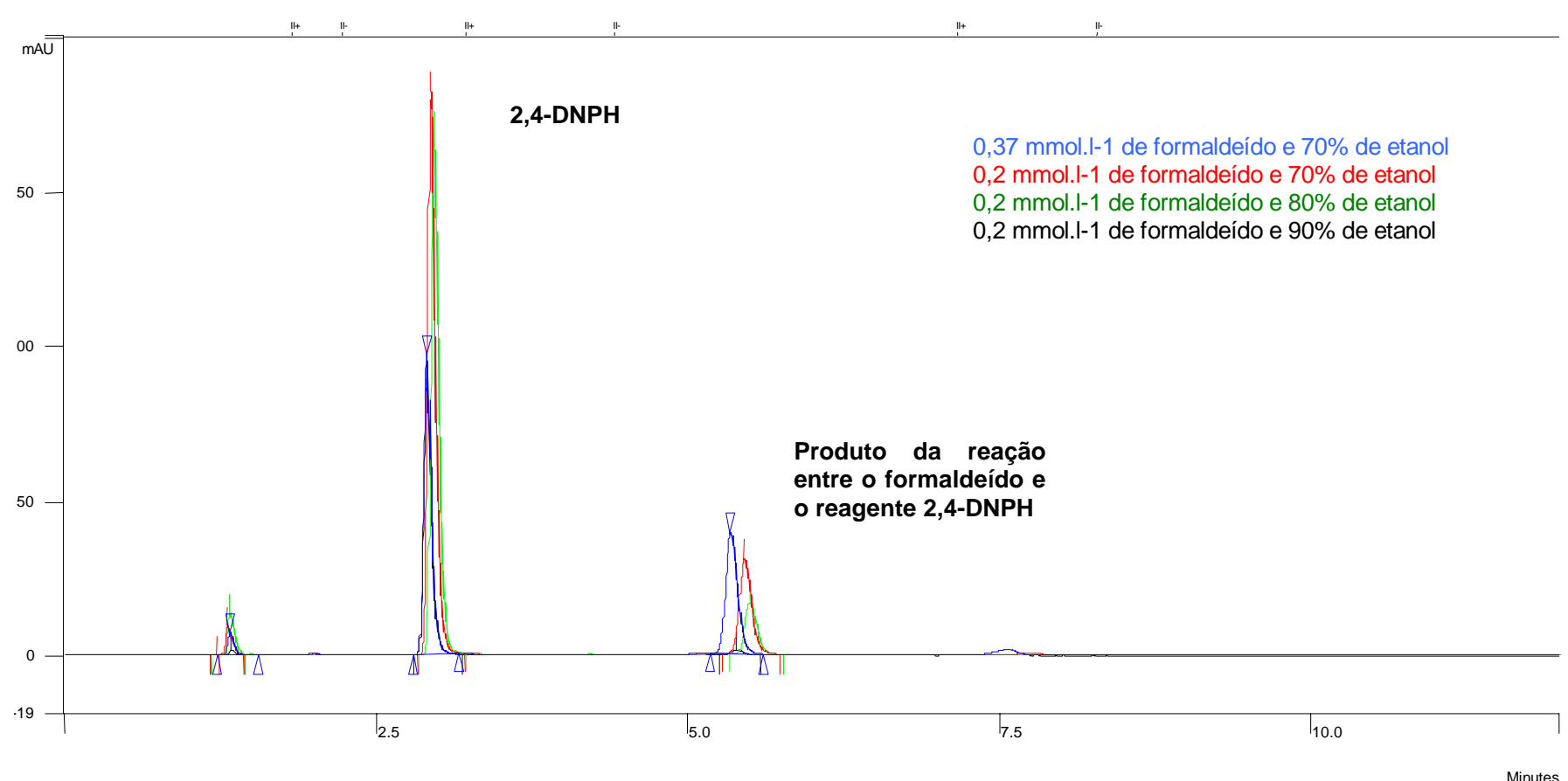

Figura 4 - Cromatogramas de amostras de formaldeído residual de cápsulas tratadas com diferentes soluções e analisadas após 24 horas de secagem: análise por HPLC em coluna Licosphere RP-18, 250x4mm, detecção ultravioleta $(\lambda=345 \mathrm{~nm})$.

\section{CONCLUSÕES}

Os resultados obtidos demonstraram que o teste de avaliação da ligação cruzada permitiu a verificação da extensão desta, sendo notavelmente influenciada pelo formaldeído e a concentração de etanol nas soluções usadas no tratamento das cápsulas. A ligação cruzada sofrida pela gelatina aumentou numa razão direta em 
Zampieri, A.L.T.C., et al./Revista Eletrônica de Farmácia Vol 2 (2), 73-79, 2005.

relação ao aumento da concentração de formaldeído contido na solução de tratamento, ao passo que, o aumento da concentração de etanol diminuiu proporcionalmente a ligação cruzada sofrida pela gelatina.

Os resultados mostraram ainda que, todas as cápsulas gastro-resistentes analisadas após 24 horas do tratamento apresentaram concentração residual de formaldeído dentro de limites aceitáveis, sendo que, o resíduo de formaldeído determinado após 30 dias de armazenamento, nas cápsulas tratadas com $0,2 \mathrm{mMol}^{-1} \mathrm{~L}^{-1}$ de formaldeído e $90 \%$ de etanol foi nulo. Vale ressaltar que, além do método cromatográfico empregado para a quantificação do formaldeído residual ser viável, apresenta vantagens sobre o método no qual foi baseado.

Portanto, a realização de ensaios que conduzam a elucidação dos diferentes parâmetros que influenciam a ligação cruzada na gelatina induzida pelo formaldeído é imprescindível para o desenvolvimento de um método de tratamento gastro-resistente eficiente e reprodutível.

\section{REFERÊNCIAS BIBLIOGRÁFICAS}

AHFS Drug information- American Hospital Formulary Service by the American Society of Health-System Pharmacists, p. 1437-1447, 1996.

BRASIL. RESOLUÇÃO DA DIRETORIA COLEGIADA N³3, Anexo 1, de 19 de abril de 2000. Dispõe sobre o regulamento técnico de Boas Práticas de Manipulação em Farmácias e seus anexos. Diário Oficial da República Federativa do Brasil, Brasília, DF, 19 de abril de 2000. Disponível em: <http://www.anvisa.gov.br>. Acesso em: junho, 2004.

BRÓJO P. A.; SOUSA B. A T. A utilização do formol no revestimento gastro-resistente de cápsulas gelatinosas duras. Boletim da Escola de Fármacia da Universidade de Coimbra, Coimbra, v. 32, p. 262-279, 1972.

FRIESS W. Collagen - Biomaterial for drug delivery. European Journal of Pharmaceutics and Biopharmaceutics, Stuttgart, v. 45, p. 113-136, 1998.

GHOLAP D.; SINGH S. The influence of drugs on gelatin cross-linking. Pharmaceutical Technology, Bethesda, v.28, n. 4, p. 94-102, 2004.

HEYDEN Y. V.; NGUYEN M. N.; DETAEVERNIER M. R.; MASSART D. L.; PLAIZIER-VERCAMMEN L.. Simultaneous determination of ketoconazole and formaldehyde in a shampoo: liquid chromatography method development and validation. Journal of Chromatography, v. 958, p. 191-201, 2002.

INTERNATIONAL PROGRAMME ON CHEMICAL SAFETY. Material data sheet, 1989, Geneva- CD-Rom.

ODDSSON E.; GUDJONSSON H.; THJODLEIFSSON B. Endoscopic findings in the stomach and duodenum after treatment with enteric-coated and plain naproxen tablets in healthy subjects. Scandinavian Journal of Gastroenterology, Oslo, v. 25, p. 231-234, 1990.

OFNER C. M.; BUBNIS W. A. Chemical and swelling evaluations of amino group crosslinking in gelatin and modified gelatin matrices. Pharmaceutical Research. New York, v. 13, n.12, 1821-1827, 1996.

PINA M. E.; SOUSA A T.; BROJO A P. Estabilização do processo de formilação de cápsulas gelatinosas. Boletim da Faculdade de Farmácia de Coimbra. Coimbra, v. 11 (1), p. 15-20, 1987.

PINA M. E.; SOUSA A T.; BROJO A P. Enteric coating of hard gelatin capsules. Part 1. Application of hydroalcoholic solutions of formaldehyde in preparation of gastro-resistant capsules. International Journal of Pharmaceutics, Amsterdam , v. 133, p.139-148, 1995.

PINA M. E.; SOUSA.; BROJO A P. Enteric coating of hard gelatin capsules. Part 2. Lioavailability of formaldehyde treated capsules. International Journal of Pharmaceutics, Amsterdam, v. 148, p. 73-84, 1996. 
Zampieri, A.L.T.C., et al./Revista Eletrônica de Farmácia Vol 2 (2), 73-79, 2005.

PRISTA L. N.; ALVES A C.; MORGADO R. Formas farmacêuticas obtidas por divisão mecânica, Cápsulas, Capítulo 6 . In: PRISTA L. N.; ALVES A C.; MORGADO R. Tecnologia Farmacêutica, Ed. Fundação Calouste Gulbenkian, 5 ed, v. 1, 1995, p. 535-553; 562-567.

RADI H.; AMIJI M. Chitosan-based gastrointestinal delivery systems. . Journal of Controlled Release, Amsterdam, v. 89, p. 151-165, 2003.

SCHACHT E.; BOGDANOV B. BUCKE V. D.; ROOZE N. Hydrogels prepared by crosslinking of elatin with dextran dialdehyde. Reactive \& Functinal Polymers, v. 33, p. 109-116, 1997.

SHEBA M.; KHEDR A.; ELSHERIEF H. Biologial and metabolic study of naproxen-propyphenazone mutual prodrug. European Journal of Pharmaceutical Sciences, Amsterdam, v. 17, p. 121-130, 2002.

THE MERCK INDEX. An Encyclopedia of Chemicals, Drugs, and Biologicals. Whitehouse Station, N J.; Thirteenth Edition, 2001, p. 778, 4261, 6445 and 8716.

TRONDSTAD R. I.; AADLAND E.; HOLLER T.; OLAUSSEN B. Gastroscopic findings after treatment with entrericcoated and plain naproxen tablets in healthy subjects. Scandinavian Journal of Gastroenterology, Oslo, v. 20, p. 239-242, 1985.

VANDELLI M. A ; RIVASI F.; GUERRA P.; FORNI F.; ARLETTI R. Gelatin microspheres crosslinked with D, Lglyceraldehyde as a potencial drug delivery system: preparation characterisation, in vitro and in vivo studies. International Journal of Pharmaceutics, Amsterdam, v. 215, p. 175-184, 2001.

VANDELLI M. A; ROMAGNOLI M.; MONTI A; GOZZI M; GUERRA P.; RIVASI F.; FORNI F. Microwave-treated gelatin microspheres as drug delivery system. Journal of Controlled Release, Amsterdam, v. 96, p. 67-84, 2004. 